\title{
Recovery from Critical COVID-19 Despite Delays in Diagnosis and Respiratory Treatment: a Cautionary Tale
}

\author{
Ouyang Fan ${ }^{1, \dagger}$, Fu Qiang ${ }^{1, \dagger}$, Guo Shuhong ${ }^{1}$, Yang Haibing ${ }^{1}$, \\ Li Xiangyang ${ }^{1}$, Tang Min ${ }^{1}$, Yang $\mathrm{Li}^{1, *}$
}

\author{
${ }^{1}$ Department of Cardiovascular \\ Medicine, Zhuzhou Central Hospital, \\ Hunan province, P. R. China \\ tThese authors contributed aqually \\ *Correspondence \\ 251957922@qq.com \\ (Yang Li)
}

\begin{abstract}
Although an acute, usually self-resolving disease, COVID-19 can also be deadly. Thus far, no approved specific treatments for this novel highly contagious disease are available, which posed great challenges on clinicians worldwide. Here we present the case of a relatively young COVID-19 patient who recovered well, despite delayed diagnosis and initiation of aggressive treatment. From the case, we speculated that: (a) Delayed diagnosis may miss the optimal antiviral treatment period for severe cases. (b) Monitoring of inflammatory markers and blood gas analysis in early stage may assist in identifying high-risk patients. (c) Glucocorticoids therapy in early stage may be harmful to the patient. (d) Once progressed to ARDS, mechanical ventilatory support should be considered as soon as possible in case of refractory hypoxemia. (e) ECMO, a scarce medical resource, should not be abused to treat COVID-19 patients with very low expected survival rates, especially during the period when medical resources are run out. (f) convalescent plasma therapy should be initiated in earlier stage of disease.
\end{abstract}

Keywords

COVID-19, Respiratory, Glucocorticoids therapy, ARDS

\section{Introduction}

A novel form of pneumonia called coronavirus disease2019 (COVID-19) [1], caused by the SARS-CoV-2 virus, has rapidly spread around the world and infected more than 5.15 million people as of 23 May 2020. Although an acute, usually self-resolving disease, COVID-19 can become severe or critical in up to $22.1 \%$ of patients [2], which is associated with a 28 -day mortality rate of $61.5 \%$ [3]. While $40 \%$ of patients who develop severe or critical disease have underlying systemic comorbidities such as cardiovascular disease, diabetes or chronic pulmonary diseases [3], reliable prediction of which COVID-19 patients will develop severe disease is currently impossible. In addition, no specific treatments are available against SARSCoV-2. This worrisome situation highlights the urgent need to detect progression to severe COVID-19 and initiate appropriate interventions as early as possible.

Here we present the case of a relatively young COVID19 patient who recovered well, despite delayed diagnosis and initiation of aggressive treatment. The course of her disease and her clinical management may provide a useful cautionary tale for clinicians struggling to treat this highly contagious disease.

\section{Case report}

A 49-year-old Chinese woman developed fever and fatigue on January 22 and presented to the local hospital the next day after having close contact with her SARSCoV-2-infected cousin, who returned from Wuhan on 19 January 2020. Her medical, surgical, allergic, and family histories are unremarkable except for a 4-year history of hyperthyroidism which was well controlled while taking Methimazole (2.5mg qd). She neither smokes nor drinks. Physical examination revealed a body temperature of 39.5 ${ }^{\circ} \mathrm{C}$, heart rate of 107 beats per minute, respiration of 19 breaths per minute, blood pressure of 138/82 $\mathrm{mm} \mathrm{Hg}$, and oxygen saturation of $98 \%$ while breathing ambient air. Lung auscultation revealed no pulmonary rales. Laboratory 
TA B L E 1. Standard value of laboratory data.

\begin{tabular}{|lcc|}
\hline Laboratory examinations & results & Reference ranges \\
\hline White-cell count & 3.94 & $(4.0-10) \times 10^{9} / \mathrm{L}$ \\
\hline Lymphocyte count & 0.61 & $0.5-0.7$ \\
\hline Red-cell count & 4.02 & $(4.0-5.0) \times 10^{12} / \mathrm{L}$ \\
\hline Platelet count & 138 & $100-300 \times 10^{9} / \mathrm{L}$ \\
\hline Erythrocyte sedimentation rate & 19 & $0-20 \mathrm{~mm} / \mathrm{H}$ \\
\hline Creactive protein & 1 & $\leq 10 \mathrm{mg} / \mathrm{L}$ \\
\hline Procalcitonin & 0.04 & $<0.5 \mathrm{ug} / \mathrm{L}$ \\
\hline Alanine aminotransferase & 14 & $0-40 \mathrm{u} / \mathrm{L}$ \\
\hline Aspartate aminotransferase & 27 & $0-40 \mathrm{u} / \mathrm{L}$ \\
\hline Total bilirubin & 7.8 & $1.71-21 \mu \mathrm{mol} / \mathrm{L}$ \\
\hline Direct Bilirubin & 3.2 & $0-3.42 \mu \mathrm{mol} / \mathrm{L}$ \\
\hline Albumin & 40.4 & $40-55 \mathrm{~g} / \mathrm{L}$ \\
\hline Globulin & 29.8 & $20-35 \mathrm{~g} / \mathrm{L}$ \\
\hline Creatine kinase & 143 & $18-198 \mathrm{u} / \mathrm{L}$ \\
\hline Creatine kinase MB & 10 & $0-25 \mathrm{u} / \mathrm{L}$ \\
\hline lactate dehydrogenase & 205 & $135.0-215.0 \mathrm{u} / \mathrm{L}$ \\
\hline Alpha hydroxy acid dehydrogenase & 151 & $90-220 \mathrm{u} / \mathrm{L}$ \\
\hline Cardiac tropnin I & 0.01 & $<0.01 \mu \mathrm{g} / \mathrm{L}$ \\
\hline Creatinine & 64.1 & $44-97 \mu \mathrm{mol} / \mathrm{L}$ \\
\hline Urea nitrogen & 4.8 & $2.9-7.14 \mathrm{mmol} / \mathrm{L}$ \\
\hline Uric acid & 285 & $89 \sim 357 \mathrm{umol} / \mathrm{L}$ \\
\hline D dimer & 0.67 & $80-500 \mathrm{ng} / \mathrm{ml}$ \\
& & \\
\hline
\end{tabular}

examinations showed decreased lymphocyte count but normal levels of $\mathrm{C}$ reactive protein (CRP) and procalcitonin, as well as normal erythrocyte sedimentation rate (ESR) (Table 1). Computed tomography (CT) of the lung showed single ground-glass opacities in the outer zone of the right lower lobe (Fig. 1). On admission, the patient was given oseltamivir (75mg bid for 5 days) as antiviral therapy, moxifloxacin ( $0.4 \mathrm{~g}$ qd for 15 days) as antibacterial therapy and diclofenac (50mg sos) as antipyretic therapy (Fig. 2).

She developed dry cough and vomiting on 25 January. laboratory reexaminations on that day showed low lymphocyte count but elevated CRP and ESR (Fig. 3). Enzyme-linked immunosorbent assays for immunoglobulin (Ig) $\mathrm{M}$ or $\mathrm{G}$ were negative for influenza $\mathrm{A}$ and $\mathrm{B}$, parainfluenza, respiratory syncytial virus, adenovirus, mycoplasma, chlamydia, rickettsia, and tuberculosis. Results for Legionella haemophilus were ambiguous. On 25 and 27 January, lung CT showed progressive increase in ground-glass opacities in multiple lobes of both lungs (Fig. 1). Given the patient's close contact with her SARSCoV-2-infected cousin, a nasopharyngeal swab specimen was taken.

While the swab sample was being processed, we administered inhaled interferon $\alpha-2 \mathrm{~b}\left(5 \times 10^{6} \mathrm{IU}\right.$ bid for 17 days) as antiviral therapy, azithromycin ( $0.5 \mathrm{~g}$ qd for 3 days) as antibacterial therapy and methylprednisolone $(80 \mathrm{mg}$ qd for 13 days) as anti-inflammatory therapy (Fig. 2). After this regime, the patient showed high fever, severe cough and dyspnea. Blood gas analysis revealed hypoxemia with arterial partial oxygen pressure $\left(\mathrm{PaO}_{2}\right)$ of $82 \mathrm{mmHg}$.

On 28 January, the patient's nasopharyngeal swab tested positive for SARS-CoV-2 based on a real-time reversetranscriptase-polymerase-chain-reaction conducted at the local Center for Disease Control and Prevention. The patient was transferred to the airborne-isolation unit at the nearest hospital approved by the government to treat COVID-19 patients

After transfer, the patient was given lopinavir/ritonavir (400mg:200mg bid for 19 days) combined with interferon $\alpha-2 \mathrm{~b}\left(5 \times 10^{6}\right.$ IU bid for 17 days $)$ as antiviral therapy, moxifloxacin ( $0.4 \mathrm{~g}$ qd for 15 days) as antibacterial therapy, ibuprofen $(0.1 \mathrm{~g}$ sos) for fever control, and nasal catheter oxygen inhalation for hypoxemia correction (Fig. 2). Body temperature returned to $37.5^{\circ} \mathrm{C}$, but she still had severe cough and dyspnea. From 29 January to 1 February, $\mathrm{PaO}_{2}$ fell from 67.7 to $48.6 \mathrm{mmHg}$; arterial oxygen saturation $\left(\mathrm{SaO}_{2}\right)$, from $94 \%$ to $88 \%$; and oxygenation index, from 322 to $162 \mathrm{mmHg}$. On 1 February, she was switched from a nasal catheter to mask for oxygen inhalation, which failed to correct hypoxemia. Lung CT on 4 February showed more than $75 \%$ of both lungs to be filled with diffuse, multiple ground-glass opacities with partial consolidation (Fig. 1). 

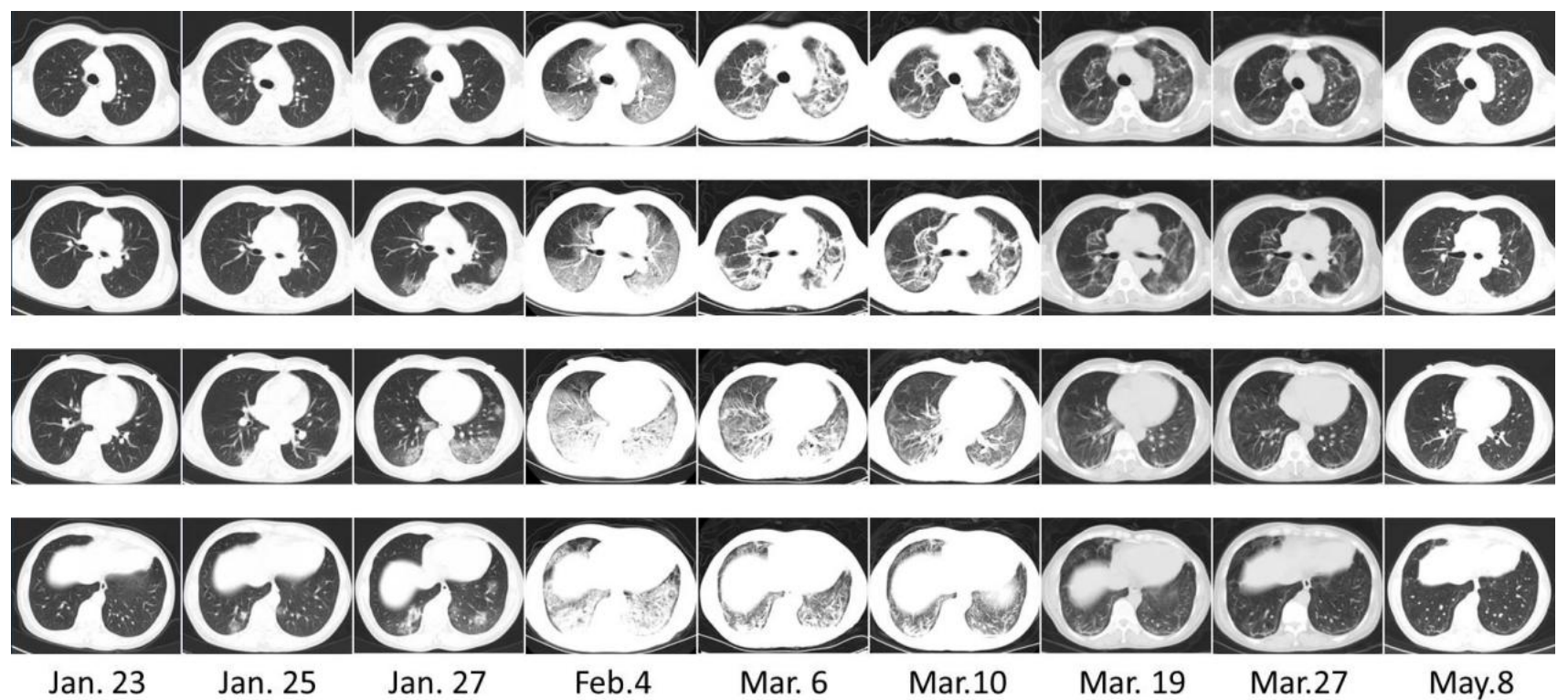

F I G U RE 1. Longitudinal series of transverse chest computed tomography images during later hospitalization. Images were taken in different course of her disease and shown in four inconsecutive slices. Single ground-glass opacities were observed in the outer zone of the right lower lobe on 23 January, which progressively increased bilaterally into multiple lobes by 27 January. Images on that day also showed a large, bilateral patch of increased density in the peripheral region of the lower lobes, with air bronchogram. On 4 February, both lungs showed large lesions manifesting consolidation and ground-glass opacities. Images on the two later dates showed substantial absorption of lesions and replacement by fibrosis, with bronchial traction and dilatation. On 19 and 27 March fibrosis lesions were gradually absorbed and almost disappeared on 5 May.

$\mathrm{PaO}_{2}$ on this day was $47.1 \mathrm{mmHg} ; \mathrm{SaO}_{2}, 86.9 \%$; and oxygenation index, $78.5 \mathrm{mmHg}$. Oxygen therapy was therefore administered by high-flow nasal cannula, but this still did not correct hypoxemia. On 7 February, the patient received non-invasive mechanical ventilation therapy, which also proved ineffective.

On 10 February, the patient was transferred to the intensive care unit, intubated tracheally and given small tidal volume positive end-expiratory pressure ventilation in $\mathrm{VC}$ mode (fraction of inspiration oxygen $\left(\mathrm{FiO}_{2}\right), 0.8$; tidal volume, $240 \mathrm{ml}$; positive end-expiratory pressure, $10 \mathrm{~cm}$ $\mathrm{H}_{2} \mathrm{O}$ ). The antibiotic regimen was switched to meropenem ( $1 \mathrm{~g} \mathrm{q} 8 \mathrm{~h}$ for 11 days) and linezolid (600mg q $12 \mathrm{~h}$ for 6 days), arbidol ( $0.2 \mathrm{~g}$ tid for 5 days) was given as antiviral therapy, and intravenous Ig (20 g qd for 4 days) was administered for immune regulation. The patient was rolled over into the prone position. Nevertheless, the oxygenation index still had not improved by 12 February, so extra-corporeal membrane oxygenation (EMCO) was started on that day $\left(\mathrm{FiO}_{2}, 0.6\right.$; pump speed, 3.5-3.8 L/min; air flow, 4-4.5 $\mathrm{L} / \mathrm{min}$ ). By 15 February, the patient's fever had subsided and her oxygenation had greatly improved.

Lavage fluid from bronchofiberscopy on 17 February tested positive for fungal spores and hyphae, and culturing revealed the presence of Candida albicans, which proved sensitive to voriconazole. This drug was administered to the patient when fever recurred on 19 February. On the same day, meropenem was replaced with piperacillin tazobactam, which was changed to daptomycin on 26 February due to concerns about ECMO-related infections.

The patient was given $200 \mathrm{ml}$ of plasma from convalescent COVID-19 patients with the neutralizing antibody above 1:640 on 19 February, then another $200 \mathrm{ml}$ on 21 February. Nasopharyngeal swab specimens taken on each of these days tested negative for SARS-COV-2. Dynamic chest X-ray suggested that pulmonary lesions were being absorbed. After a spontaneous breathing test on 23 February, the ventilator was withdrawn and endotracheal tube removed. The patient was given $100 \mathrm{ml}\left(10^{6} / \mathrm{ml}\right)$ of stem cells from umbilical cord blood on 26 February, and another $100 \mathrm{ml}$ on 29 February.

By this time, the patient showed normal heart, liver and kidney function, all her symptoms had been relieved, and her lymphocyte count and CRP had returned to normal. ECMO was discontinued on 2 March, and antibiotic therapy was stopped on 6 March. Lung CT on 6 and 10 March showed that most ground-glass opacities had been absorbed and the lesions had been largely replaced by fibrosis with bronchial traction and dilatation (Fig. 1).

The patient was provided psychological counseling, pulmonary rehabilitation exercises, and assisted ambulation, and she was encouraged to eat. Her oxygen saturation remained above $95 \%$ during low-flow oxygen inhalation. She was discharged on 14 March. During follow-up to date, Lung CT showed that fibrosis lesions were gradually absorbed on 19 and 27 March and almost disappeared on 5 May. Her lung function on 5 May was normal. She could tolerate normal daily activities. 
A

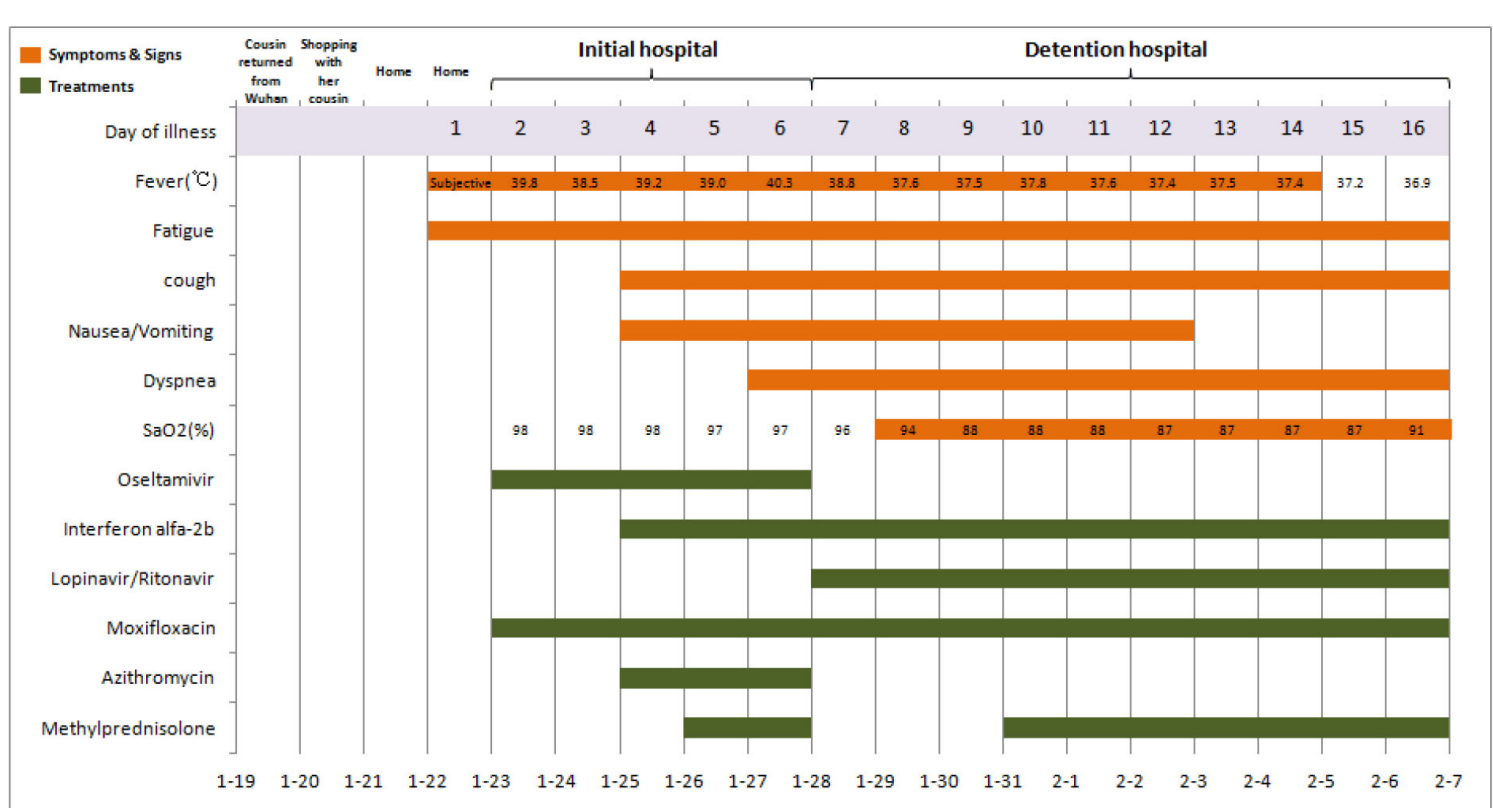

B

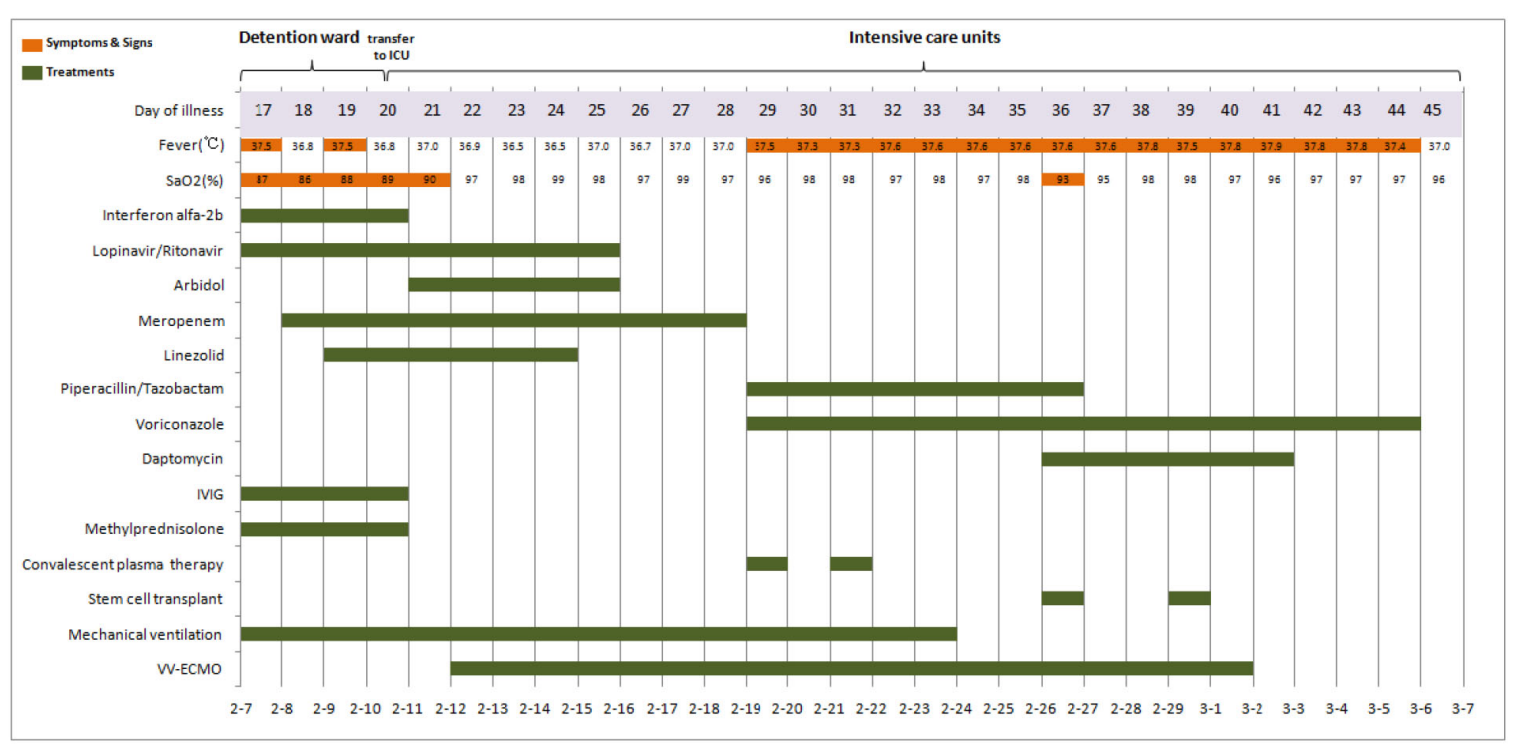

\section{F I G U R E 2. Timeline of disease course according to days from initial presentation of illness.}

\section{Discussion}

Within only 6 days of onset of COVID-19 symptoms, our patient progressed to hypoxemia and acute respiratory distress syndrome (ARDS), necessitating mechanical ventilation and ECMO support. Given her low lymphocyte count and sharp increase in CRP level, we attribute this rapid progression to a cytokine storm triggered by massive viral replication [4, 5]. To counteract this storm, we gave the patient methylprednisolone; this or other glucocorticoids are often used to inhibit such storms in patients infected with bacteria or virus. This early glucocorticoid therapy may have contributed to our patient's rapid progression. A systematic review concluded that low- to moderate-dose glucocorticoids do not affect mortality among patients with
H7N9 pneumonia and actually increase mortality among patients with $\mathrm{H} 1 \mathrm{~N} 1$ pneumonia without respiratory failure [6]. In addition, early corticosteroid treatment is associated with higher and more persistent viral load in patients with mild pneumonia associated with severe acute respiratory syndrome (SARS) or Middle East respiratory syndrome (MERS) [7, 8]. Future studies should examine whether glucocorticoid therapy is at all appropriate for COVID-19 patients.

Another factor that may have contributed to our patient's worsening disease was that she was not shifted to mechanical ventilation until 10 days after ARDS onset, even though her oxygenation index was below $150 \mathrm{mmHg}$. This late intervention may help explain why our patient ultimately required ECMO. Diffuse alveolar damage with cellular 


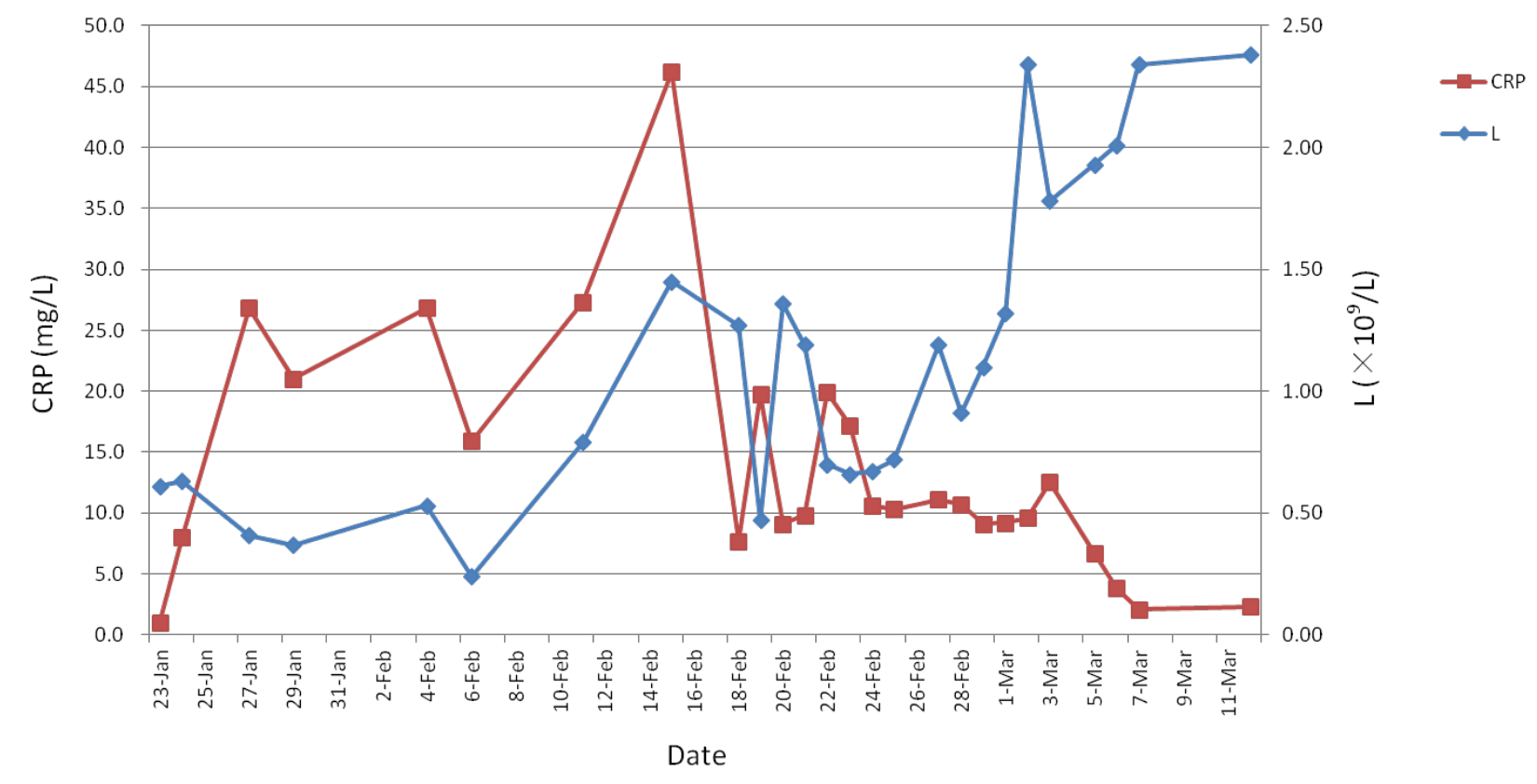

F I G U R E 3. Longitudinal characteristics of lymphocytes counts and C reactive protein in the peripheral blood.

fibromyxoid exudates was observed in the lungs of a 50year-old man who died of severe ARDS caused by SARS$\mathrm{CoV}-2$ infection and who showed hyperactivation of $\mathrm{CD}^{4+}$ and $\mathrm{CD}^{8+} \mathrm{T}$ cells as well as strong expression of granulysin and perforin [9]. A strong pulmonary exudative response was also observed in an 85-year-old man with COVID-19 [10]. These previous reports and the present case suggest that patients showing hypoxia, viral damage and cytokine storm should be intubated as early as possible and given mechanical ventilation, supplemented by sputum suction and medical expectorant treatment.

Identifying progression to severe COVID-19 as early as possible is critical given that the disease can progress from first symptom to dyspnea and then to ARDS in about one week [11]. Our patient showed persistent low lymphocyte count, a sharp increase in CRP (Fig. 3) and progressive increase in ground-glass opacity in multiple lobes of both lungs (Fig. 1) within a few days after symptom onset. These may be early warning signs of progression to severe COVID-19. Routine arterial blood gas monitoring may be necessary for patients with these early warning indicators, given that fingertip pulse oximetry may fail to detect hypoxemia early enough because of pulmonary compensation. Our patient showed $97 \%$ oxygenation by pulse oximetry when she first developed dyspnea, but her $\mathrm{PaO}_{2}$ had already fallen to $82 \mathrm{mmHg}$.

Our patient survived with the help of ECMO, but one study suggests that only a fraction of COVID-19 patients on ECMO survive [2]. Prognosis after ECMO depends on many factors such as age, underlying diseases, severity of COVID-19 disease, complications, and concomitant therapies. Therefore, prognostic scoring tools such as the Respiratory ECMO Survival Prediction (RESP) score [12] should be used before ECMO to determine whether the patient is likely to benefit. Our patient had a RESP score of 3, suggesting high likelihood of survival after ECMO.

Our case illustrates the need for diagnosis of COVID19 as soon as possible to ensure timely antiviral treatment, and the usefulness of monitoring inflammatory markers and blood gases early in the disease in order to identify patients at high risk of severe progression. Mechanical ventilation should be initiated as soon as possible if the patient has refractory hypoxemia. It may be advisable to avoid glucocorticoid therapy for patients who experience an inflammatory storm, which should be investigated in prospective studies. ECMO can be effective for treating severe and critical COVID-19, but it may be advisable to reserve this scarce resource for patients with strong changes of survival.

Although convalescent plasma from cured COVID-19 patients was transfused to our patient, it may not really work. Meanwhile, the risk of transfusion-transmitted infections, such as human immunodeficiency virus, hepatitis $B$ virus, hepatitis $C$ virus and syphilis, should not be neglected [13]. Hence, it may be unnecessary to administrate convalescent plasma in the late stage of the disease.

\section{ACKNOWLEDGEMENTS}

First and foremost, I appreciate my country who give me a comfortable learning atmosphere. Second, my sincere appreciation also goes to all my colleagues, who are my proud of my life. Last but not least, I want to thank all my friends, for their encouragement and support, without their enlightening instruction, impressive kindness and patience, I could not have completed my manuscript. 


\section{CONFLICT OF INTEREST}

We declare that we do not have any commercial or associative interest that represents a conflict of interest in connection with the work submitted.

\section{REFERENCES}

[1] Wu F, Zhao S, Yu B, et al. A new coronavirus associated with human respiratory disease in China. Nature. 2020;579:265-269.

[2] Pan A, Liu L, Wang C, et al. Association of Public Health Interventions With the Epidemiology of the COVID-19 Outbreak in Wuhan, China. JAMA. Published online April 10, 2020. doi:10.1001/jama.2020.6130.

[3] Yang X, Yu Y, Xu J, et al. Clinical course and outcomes of critically ill patients with SARS-CoV-2 pneumonia in Wuhan, China: a singlecentered, retrospective, observational study. Lancet Resp Med. 2020.

[4] Bhatia M, Moochhala S. Role of inflammatory mediators in the pathophysiology of acute respiratory distress syndrome. J Pathol. 2004;202:145-156.

[5] Meduri G U, Headley S, Kohler G, et al. Persistent elevation of inflammatory cytokines predicts a poor outcome in ARDS: plasma IL-1 $\beta$ and IL-6 levels are consistent and efficient predictors of outcome over time. Chest. 1995;107:1062-1073.

[6] Cao B, Gao H, Zhou B, et al. Adjuvant corticosteroid treatment in adults with influenza A (H7N9) viral pneumonia. Crit Care Med 2016;44:e318-e328.

[7] Lee N, Chan K C A, Hui D S, et al. Effects of early corticosteroid treatment on plasma SARS-associated Coronavirus RNA concentrations in adult patients. J Clin Virol. 2004;31:304-309.

[8] Arabi Y M, Mandourah Y, Al-Hameed F, et al. Corticosteroid therapy for critically ill patients with Middle East respiratory syndrome. Am J Resp Crit Care. 2018;197:757-767.

[9] Xu Z, Shi L, Wang Y, et al. Pathological findings of COVID-19 associated with acute respiratory distress syndrome. Lancet Resp Med. 2020

[10] Liu Qian, Wang Rongshuai, Qu Guoqiang. Report of gross autopsy findings of a dead COVID-19 patient. J Forensic Sci. 2020;36:19-21.

[11] Wang D, Hu B, Hu C, et al. Clinical characteristics of 138 hospitalized patients with 2019 novel coronavirus-infected pneumonia in Wuhan, China. JAMA. 2020

[12] Schmidt M, Bailey M, Sheldrake J, et al. Predicting survival after extracorporeal membrane oxygenation for severe acute respiratory failure. The Respiratory Extracorporeal Membrane Oxygenation Survival Prediction (RESP) score. Am J Resp Crit Care. 2014;189:13741382.

[13] Maclennan S, Barbara J A. Risks and side effects of therapy with plasma and plasma fractions. Best Pract Res Cl Ha. 2006;19:169-189.

How to cite this article: Ouyang Fan, Fu Qiang, Guo Shuhong, Yang Haibing, Li Xiangyang, Tang Min, Yang Li. Recovery from Critical COVID-19 Despite Delays in Diagnosis and Respiratory Treatment: a Cautionary Tale. Signa Vitae. 2020;16(1):193-198. doi: $10.22514 /$ sv.2020.16.0027. 